\title{
Identification of Whirling Motions by Phase Unwrapping Technique
}

Rong-Juin Shyu

Department of Naval Architecture National Taiwan Ocean University Keelung, Taiwan

Follow this and additional works at: https://jmstt.ntou.edu.tw/journal

Part of the Engineering Commons

\section{Recommended Citation}

Shyu, Rong-Juin (1993) "Identification of Whirling Motions by Phase Unwrapping Technique," Journal of Marine Science and Technology. Vol. 1: Iss. 1, Article 3.

DOI: $10.51400 / 2709-6998.2473$

Available at: https://jmstt.ntou.edu.tw/journal/vol1/iss1/3

This Research Article is brought to you for free and open access by Journal of Marine Science and Technology. It has been accepted for inclusion in Journal of Marine Science and Technology by an authorized editor of Journal of Marine Science and Technology. 


\title{
IDENTIFICATION OF WHIRLING MOTIONS BY PHASE UNWRAPPING TECHNIQUE
}

\author{
Rong-Juin Shyu \\ Department of Naval Architecture \\ National Taiwan Ocean University \\ Keelung, Taiwan
}

Key words: whirling, rotating shaft, phase unwrapping, strain gages.

\begin{abstract}
A method for identifying the whirling motion of a rotating shaft has been developed. This method is based on the phase unwrapping of two strain gages' signals. These two stain gages are being attached ninety degrees apart onto the rotating shaft thus observe the shaft's motion in a rotating coordinate with speed the same as that of the shaft. With such arrangement of the strain gages, the whirling motions of the shaft can be identified provided that the rotation speed of the shaft is known. These whirling motions include forward whirl and backward whirl. Equations are given to show how to interpret the strain measurements. Examples from an experiment of a vertical rotating shaft are also given to show the use of this method. The proposed method is shown to be able to identify the whirling motions, which can not be achieved using only proximity probes.
\end{abstract}

\section{INTRODUCTION}

Shaft systems exist in many industrial applications, for examples, turbines, compressors, propeller systems, etc. These systems usually operate in rotating conditions. Due to mass eccentricity, interactions with journal bearings, the shaft circles around the originally undeflected center line of the shaft as the shaft starts to rotate. This motion is called whirling and can be roughly divided into two categories. One is the forward whirl and the other is the backward whirl. The shaft is said to be in the forward whirl if the direction of rotation and the whirling is the same, and backward whirl if the direction is opposite. Figure 1 shows the cross section of a shaft and the directions of the whirling motions. These whirling motions are common to a shaft system and are potentially hazardous to a shaft system. Large amplitude forward whirl usually occurs when the rotation speeds are close to one of the shaft's bending natural frequencies. This may cause contacts between shaft and the casings, and result in the wear in the surface of the shaft. Backward whirl is usually caused by the friction between shaft and the casing or journal bearing, and the backward whirl motion starts when the perturbed velocity of the shaft exceeds a threshold value[1]. This motion effectively increases the stress variations per unit time,

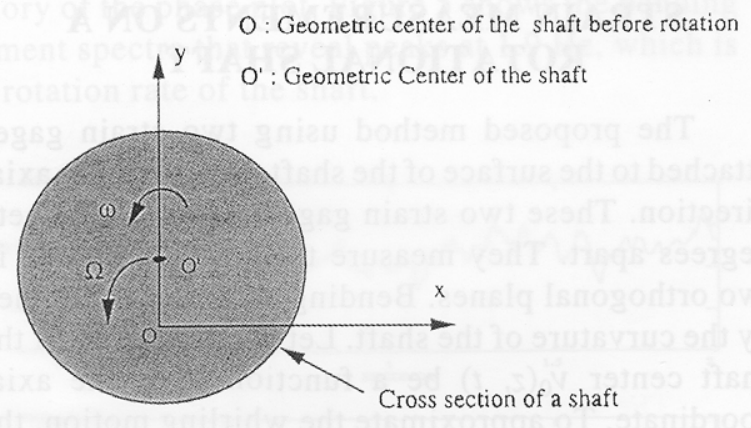

$\omega$ : Rotation speed of the shaft with respect to point $\mathrm{O}^{\prime}$

$\Omega$ : Rotation speed of point $\mathrm{O}^{\prime}$ with respect to point $\mathrm{O}$

Oxy ; A stationary reference coordinate

Fig. 1. Whirl Motions of the Shaft. 
thus, reduces the fatigue life of the shaft system. Therefore, methods for identifying their existence are needed to take preventive measures.

The conventional methods for identifying shaft's motion involve the use of proximity probes[2]. The proximity probe can be either of mechanical or magnetic type that is usually being installed onto the casing of the shaft, and measures the surface displacement of the shaft. They usually come in pairs and are being separated ninety degrees apart. The motion of the shaft's center with respect to a stationary reference frame can be identified with this device. However, the motion at other points on the shaft can not be revealed unless the relative speed of shaft to the shaft's center is known. Therefore, no information regarding the whirl motions of the shaft can be obtained using this method.

This paper proposed a method for identifying the whirling motions using strain gages attached to the shaft system. The previous usages of strain gages in determining shaft's motion limited only to the torsional vibration measurement, where the torsional fluctuating signal is measured by circumferential strain gages attached onto the shaft's surface[2]. Here, instead a two strain gages system was used to measure the bending strains on two orthogonal planes. The strain gages are attached to the surface of the shaft at the same cross section and rotating with the shaft, therefore measure the relative motion of the shaft to its center, and thereby reveal the motion at every points on the shaft. Because the strain gages are rotating with the shaft, a device called slip ring is used to carry the strain signals to a recording unit. Other device like radio link is also used frequently in such experiment to transmit signal from a rotating object. Finally, a signal processing technique is also being proposed to extract whirling information from such measurements.

\section{STRAIN MEASUREMENTS ON A ROTATIONAL SHAFT}

The proposed method using two strain gages attached to the surface of the shaft parallel to the axial direction. These two strain gages are exactly ninety degrees apart. They measure the bending strains in two orthogonal planes. Bending strain is determined by the curvature of the shaft. Let the deflection of the shaft center $v_{0}(z, t)$ be a function of $z$, the axial coordinate. To approximate the whirling motion, the deflection shape of half a sine wave is assumed; that is

$$
v_{0}(z, t)=R \sin \left(\frac{\pi_{z}}{L}\right) F^{\prime}(t)
$$

where $R$ is the whirling amplitude of the shaft, $L$ the length of the shaft and $F(t)$ a function of time. The bending moment corresponding to $v_{0}(z, t)$ is given by the moment-curvature relationship

$$
B(z, t)=E I \cdot \frac{d^{2} v_{0}}{d z^{2}}=-R \frac{E I \pi^{2}}{L^{2}} \sin \left(\frac{\pi z}{L}\right) F(t)
$$

where $E$ is the modulus of elasticity and $I$ the moment of inertia of the shaft. The bending moment $B(z, t)$ was measured at a single location on the shaft's surface by two orthogonally oriented strain gage bridges. These bridges yielded the two vector components $B_{x^{\prime}}(t)$ and $B_{y^{\prime}}(t)$. The measured bending moment is then a vector in an orthogonal $x^{\prime} y^{\prime} z^{\prime}$ coordinate system, attached to the center line of the undeflected shaft and rotating with the shaft at $\omega$, which is the rotation speed of the shaft. The coordinate systems, both stationary and rotating, are defined in Figure 2. The bending moments measured at rotating coordinate are

$$
\begin{gathered}
\vec{B}(t)=B^{\prime}(t) \vec{i}+B^{\prime}(t) \vec{j} \\
\vec{B}(t)=|B|\left[\cos (\phi(t)) \vec{i}+\sin (\phi(t)) \overrightarrow{j^{\prime}}\right]
\end{gathered}
$$

where $\phi(t)$ is the angle between moment vector, $\vec{B}(t)$, and $y^{\prime}$ axis of the rotating coordinate system and $\vec{i}$ and $\vec{j}$ are unit vectors in the $x^{\prime}$ and $y^{\prime}$ directions. If the shaft is rotating at $w$ arıd whirling at $\Omega$ speed, then equation (4) may be expressed as below:

$$
\vec{B}(t)=|B|[\cos ((\omega-\Omega) t) \vec{i}+\sin ((\omega-\Omega) t) \vec{j}]
$$

The rotating $O^{\prime} x^{\prime} y^{\prime}$ coordinate system representation is particularly useful, because it represents the bending moment time history that is experienced by the shaft and is therefore a measure of the stress time history which is relevant to cyclic fatigue damage. An important feature of this expression is that the bending moment, and hence the bending stresses, vary at

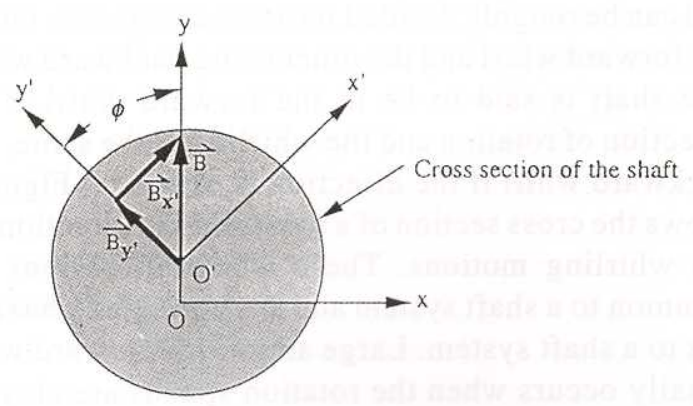

$O^{\prime} x^{\prime} y^{\prime}$ : A rotating coordinate fixed to the center of the shaft

Fig. 2. Coordinate System, Bending Moment and Phase Angle Definition. 
the difference between the shaft rotation rate, $\omega$, and the whirl rate, $\Omega$. The difference frequency is the frequency of stress cycles which would be experienced by the shaft under whirling conditions.

Equation (5) is valid for all whirling conditions, One must only evaluate the magnitude and correct sign (plus for clockwise rotation and minus for counterclockwise rotation) of the shaft rotation and whirl rates. The phase angle, $\phi(t)$ can be estimated from $B_{x^{\prime}}(\mathrm{I})$ and $B_{y^{\prime}}(t)$, the independently measured bending moment components, as follows:

$$
\begin{gathered}
\phi(t)=\tan ^{-1}\left[\frac{B_{y^{\prime}}(t)}{B_{x^{\prime}}(t)}\right]+\phi_{0} \\
=\tan ^{-1}\left[\frac{\sin ((\omega-\Omega) t)}{\cos ((\omega-\Omega) t)}\right]+\phi_{0} \\
=(\omega-\Omega) t+\phi_{0} \\
\frac{d \phi}{d t}=(\omega-\Omega)
\end{gathered}
$$

where $\phi_{0}$ is a constant depending on initial conditions. Therefore, from two independent and orthogonal bending moment measurements the difference between the whirl rate and rotation rate can be determined. The slope of the $\phi(t)$ plot, which from equation (7), is the difference frequency $(\omega-\Omega)$. Since the rotation rate $\omega$ is usually known from a rotational encoder, the whirl rate $\Omega$ can then be deduced.

\section{PHASE UNWRAPPING}

The phase between $B_{x^{\prime}}$ and $B_{y^{\prime}}$, as mentioned above, is useful in revealing the motion of the shaft. The technique used in presenting phase angle is called phase unwrapping. The term 'unwrapped' phase angle refers to the way the arctangent function is computed from real data. The arctangent function used here is not restricted to a range of $-\pi$ to $+\pi$. Rather, the phase angle is meant to accumulate linearly with time as shown in equation (6). The use of phase unwrapping first appeared in the computation of complex cepstrum, where a continuous phase information is needed to obtain a unique transformation between time history and the associated cepstrum [3]. The algorithm is slightly modified here for the computation for the phase between $B_{x^{\prime}}(t)$ and $B_{y^{\prime}}(t)$ signals. Consider the function arctangent

$$
\phi(t)=\tan ^{-1}(g(t))
$$

where $g(t)$ is any given time function. The principal values of the arctangent range from -180 to $180 \mathrm{de}-$ grees. Therefore, if $\phi(t)$ is plotted, discontinuities may occur. Phase unwrapping is a method to make $\phi(t)$ continuous. One way to achieve phase unwrapping is presented in the following steps, assuming that $\phi_{i+1}$ and $\phi_{i}$ are two adjacent points in time, the procedure involves

1. Determine the absolute value of $f_{i+1}-f_{i}$, if this value is greater than 180 degrees, then do the phase unwrapping, otherwise proceed to the next point.

2. Determine the sign of $f_{i+1}-f_{i}$, if phase unwrap ping is needed.

3. If the sign is plus, subtract 360 degrees from every point after $f_{i}$; if the sign is minus, add 360 degrees to every point after $f_{i}$.

By performing the above procedure for every data point, one can obtain the unwrapped phase plot of the function, arctangent.

\section{EXAMPLES}

Actual data was collected during an experiment on a vertical shaft rotating in a hole. Two orthogonal strain gages are being attached to the shaft. Their signals are connected to a stationary recording system via a slip ring. Two cases are discussed; they are forward synchronous and backward whirl.

Figure 3 shows the time histories for the bending moments of the first case and Figure 4 shows the unwrapped phase of the two strain gages signal. The rotary speed of the shaft was at $116 \mathrm{rpm}$. The time history for the unwrapped phase angle, shown in Figure 4, reveals that the mean phase angle remained constant. Vibration, not whirling, is revealed as additional fluctuations in phase angle about the mean. The interpretation for this case is that the zero average phase rate shows that the shaft was exhibiting forward synchronous whirl. Forward synchronous whirl seen from a rotating coordinate is a static deflection of the shaft, therefore, no average phase variation in the time history of the phase plot. Figure 5 shows the bending moment spectra that reveal peaks at $1.9 \mathrm{~Hz}$, which is the rotation rate of the shaft.
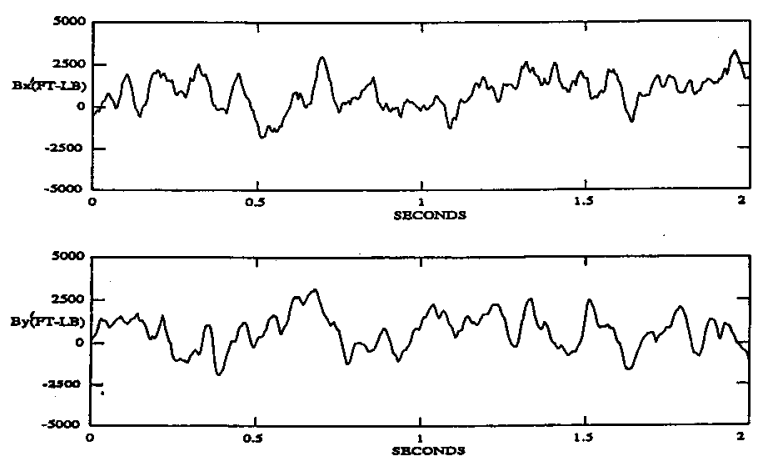

Fig. 3. Time History of Phase Angle for Forward Synchronous Whirl. 


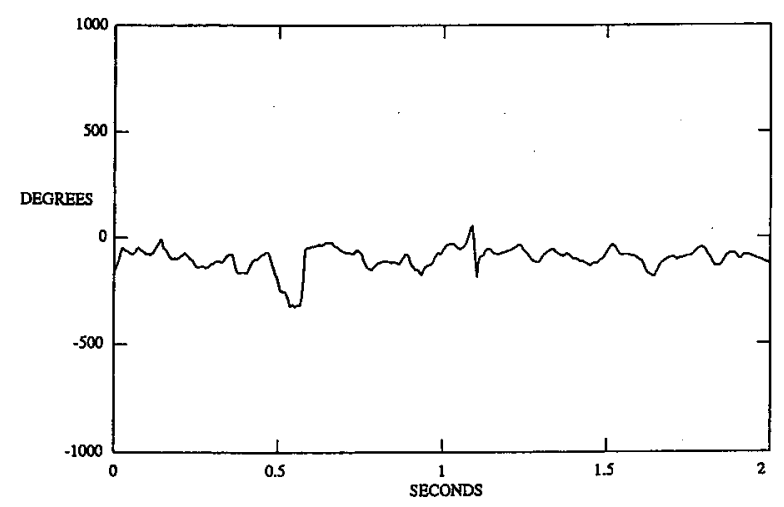

Fig. 4. Time History of Bending Moments for Backward Whirl.
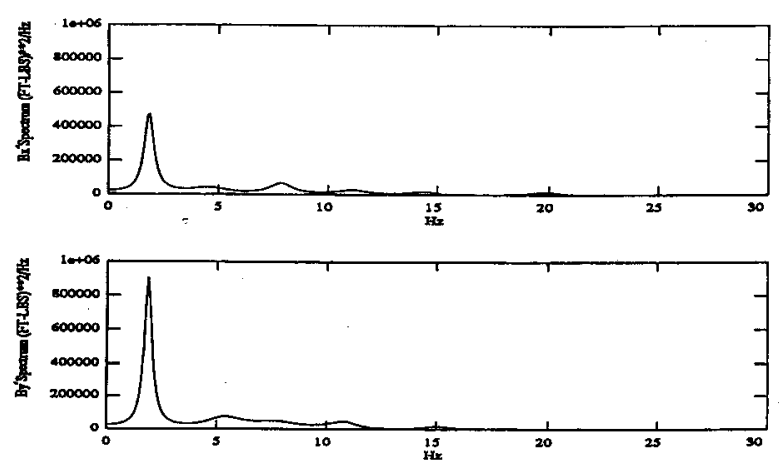

Fig. 5. Bending Moment Spectra for Forward Synchronous Whirl.

Figure 6 shows the time history for the bending moments of the second case and Figure 7 shows the phase angle. The rotation speed $\omega$ was $60 \mathrm{rpm}(1.0 \mathrm{~Hz})$ in this case. Figure 7 shows that the mean phase rate was 1200 degrees $/ \mathrm{sec}(3.33 \mathrm{~Hz})$. Thus, the difference frequency, $(\omega-\Omega) / 2 \pi$, was $3.33 \mathrm{~Hz}$. This is verified by the location of the peaks of the bending moment spectra, shown in Figure 8. Requiring $\omega / 2 \pi$ to be 1.0 $\mathrm{Hz}$. results in an estimate for $\Omega / 2 \pi$ of $-2.33 \mathrm{~Hz}$. Therefore, the shaft is whirling backward at this frequency. The stress cycle increases under this circumstance as shown in the spectral peak in the bending signal. As the stress cycle increases, the fatigue life of the shaft will be reduced. It is potentially hazardous to the operation of a shaft system.

\section{CONCLUSIONS}

The motion of a rotating shaft has been successfully identified using the proposed method provided that the rotation speed of the shaft is known. Strain gages, under this circumstance, must being attached to the shaft and the signal must be transmitted to a stationary recording unit using slip rings or radio links. It has the advantages over the proximity probes, which measures only the orbit of the shaft's center,
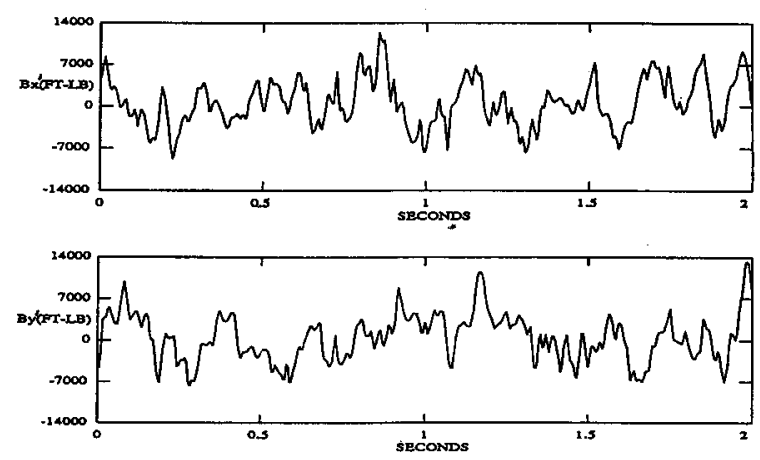

Fig. 6. Time History of Bending Moments for Backward Whirl.

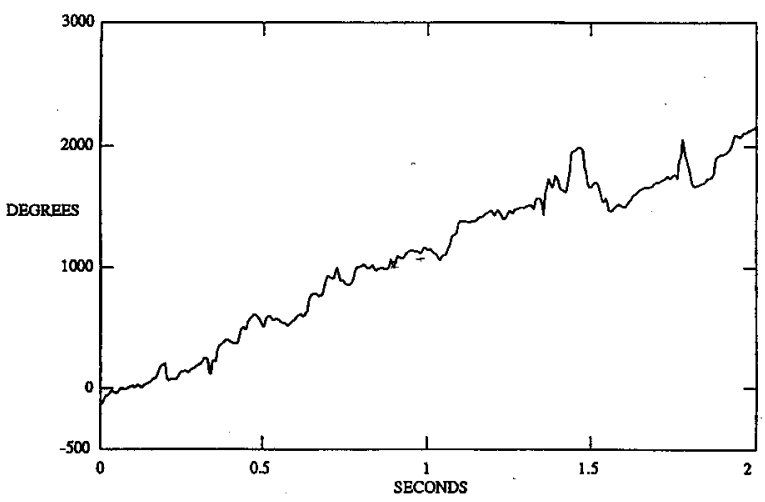

Fig. 7. Time History of Phase Angle for Backward Whirl.
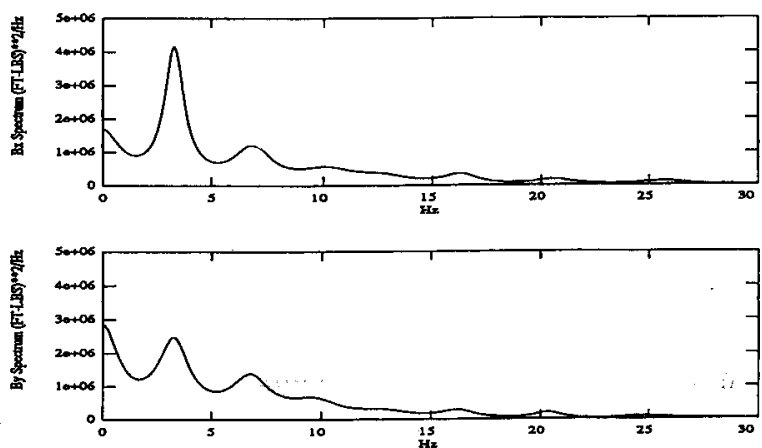

Fig. 8. Bending Moment Spectra for Backward Whirl.

and no information regarding to the forward or backward of the shaft can be revealed. The proposed method can therefore be used to monitoring the shaft systems for potential indications of the shaft's failures.

According to the method proposed above, if the phase information from the strain gage's signal is constant, it is very likely that the shaft is exhibiting synchronous whirl and the shaft is sliding along the circumference of the bearings and wear against the journal bearings. Whereas, if the shaft is exhibiting backward whirl, it would roll along the circumference 
of the bearings, the phase would thus increase at a rate equal to the sum of the rotation and the whirl speed. This motion tends to increase the stress cycles and may reduce the fatigue life of the shaft system. If the motion of the shaft is partially sliding and partially rolling, the whirl rate can also be identified if the rotation speed is known. Therefore, an on-line tool for monitoring the shaft's motion can be provided by this method.

\section{REFERENCES}

1. Zhang, W., Dynamic Instability of Multi-Degreeof-Freedom Flexible Rotor System Due to Full Annular Rub. In it Proceedings of the International Conference on Vibrations in Rotating Machinery, Edinburgh, England, 305-310, 1988.

2. Mitchell, J. S., it Machinery Analysis and Monitor ing. 26-80, PennWell Publishing Company, Tusla, Oklahoma, U.S.A., 1981.

3. Tribolet, J. M., "A New Phase Unwrapping Algo rithm." IEEE Transactions on Acoustics, Speech and Signal Processing, ASSP-25(2), 170-177, April 1977. 